Y. MUTO

KODAI MATH. J.

6 (1983), 308-332

\title{
SOME PROPERTIES OF ISOMETRIC MINIMAL IMMERSIONS OF SPHERES INTO SPHERES
}

\author{
By Yosio Mutō
}

\section{Introduction.}

The purpose of the present paper is to study isometric minimal immersions $f: S^{m} \rightarrow S^{n-1}$ where each sphere is endowed with some Riemannian metric of constant curvature. For that purpose we use, besides eigenfunctions of the Laplacian $\Delta$ on $S^{m}$, some tensors in $R^{m+1}$. It is known that such immersions are classified by a natural number $s$ which is called the order. When $m$ and $s$ are given, we can take $n$ at haphazard. If $n$ is too large, all immersions $f$ are not full. Hence we choose $n$ so that most of the immersions $f$ are full. The method employed in the present paper is expected to be useful in the study of some geometric properties of the image $f\left(S^{m}\right)$.

Isometric minimal immersions $f: S^{m} \rightarrow S^{n-1}$ of spheres into spheres were studied by M.P. do Carmo and N.R. Wallach [1]. From their results we see that there exist three kinds of isometric minimal immersions, namely, standard minimal immersions, non standard full isometric minimal immersions and non full isometric minimal immersions. Any stardard minimal immersion is a full immersion and, when $s$ is given, any two standard minimal immersions $\phi_{1}$ and $\phi_{2}$ of order $s$ are equivalent to each other in the sense that there exists an isometry of $S^{n-1}$ which sends $\psi_{1}\left(S^{m}\right)$ into $\psi_{2}\left(S^{m}\right)$. In the present paper, $S^{m}$ is endowed with the standard Riemannian metric $g$ so that $S^{m}$ can be considered as a unit hypersphere of $R^{m+1}$ and $S^{n-1}$ is considered as a hypersphere of radius $r$ in $R^{n}$ with 0 as the center. $n$ is given by

$$
n=(2 s+m-1) \frac{(s+m-2) !}{s !(m-1) !}
$$

and $r$ is given by

$$
r^{2}=\frac{m}{s(s+m-1)}
$$

We omit the case $s=1$ as a too simple case. We also see in [1] that the set of equivalence classes of isometric minimal immersions for $s>3$ is parametrized by a compact convex body $L$ in a certain vector space $W_{2}$ if $m \geqq 3$. The interior

Received October 23, 1982 
points of $L$ correspond to the equivalence classes of full isometric minimal immersions and the boundary points of $L$ correspond to those of non full isometric minimal immersions. If $s \leqq 3$, then there exist only standard minimal immersions.

Properties of isometric minimal immersions of spheres into spheres are being investigated by $\mathrm{K}$. Tsukada [10] and K. Mashimo [3] in various aspects. In the present paper some other aspects are treated. It presents some fundamental formulas in tensor form which wait for various applications. As examples of such applications, some properties of the image, which is considered as a submanifold of the Euclidean space $R^{n}$ containing the target sphere $S^{n-1}$, are studied in connection with the Gauss map.

It is known that there exists an intimate relation between eigenfunctions of the Laplacian $\Delta$ on $S^{m}$ and an isometric minimal immersion of $S^{m}$ into $S^{n-1}$ [9]. Hence in $\S 2$ we consider immersions $f: S^{m} \rightarrow S^{n-1}$ brought about by eigenfunctions of order $s$. For such an immersion which we call an immersion of order $s$, we have a tensor of $R^{m+1}$ of degree $2 s$, which is called the tensor of degree 2s associated with the immersion $f$. Since $S^{m}$ is considered as a unit hypersphere of $R^{m+1}$, the position vector $u$ of a point $p$ of $R^{m+1}$ ranging $S^{m}$ plays the central role. Thus in $\S 3$ such tensors are studied for isometric minimal immersions. We prove there that the isometric minimal immersions of order $s \leqq 3$ are rigid, which is in fact already known [1]. A space of tensors of degree $2 s$ which we denote by $B_{s, s}$ plays an important role in the theory of minimal immersions. The unit element of $B_{s, s}$ is studied in $\S 4$. In $\S 5$ standard minimal immersions are studied. The tensor of degree $2 s$ associated with the standard minimal immersions of order $s$ is proved to be the unit element of $B_{s, s}$ multiplied by a certain number. In $\S 6$ the relation between an arbitrary isometric minimal immersion and a standard minimal immersion, both of order $s$, is studied and it is shown that any isometric minimal immersion is characterized by a tensor $\hat{d}_{s, s}$ of degree $2 s$ and an interval $I$ depending on $\hat{d}_{s, s}$. This tensor vanishes if $s \leqq 3$ and depends on at least 18 independent variables if $s=4$ and $m=3$. This fact has been pointed out by do Carmo and Wallach [1]. All properties of isometric minimal immersions are printed in the tensor $\hat{d}_{s, s}$ but unfortunately its computation is perhaps impracticable except for small $m, m \geqq 3$, and $s, s \geqq 4$. In $\S 7$ we return to fundamental formulas given in $\S 3$ and deduce some properties of isometric minimal immersions.

The present study was stimulated by the paper of M.P. do Carmo and N.R. Wallach [1] and some papers of H. Nakagawa, K. Ogiue and T. Itoh [2], [7]. Thus some of the results due to do Carmo and Wallach reappear in the present paper bearing different appearance. But the method employed here will be of some use in differential geometry of such minimal immersions as treated in the present paper. Most of the obtained results are contained in Theorems 3.3, 4.1, $5.2,5.4,6.2,6.3,7.1,7.3,7.4$ and Lemmas 3.1, 5.1.

The author wishes to express his hearty thanks to Prof. K. Ogiue for valuable suggestions. The author is also grateful to the referee for his kind advice in improving the paper. 


\section{Eigenfunctions of the Laplacian and somettensors of $R^{m+1}$.}

In the present paper we use indices as follows :

$$
\begin{aligned}
& A, B, C, \cdots=1, \cdots, n, \\
& a, b, c, \cdots, h, i, j, \cdots=1, \cdots, m+1, \\
& \kappa, \lambda, \mu, \cdots=1, \cdots, m,
\end{aligned}
$$

and adopt the usual summation convention if possible. Let $u^{1}, \cdots, u^{m+1}$ be functions on $S^{m}$ such that, for each point $p$ of $R^{m+1}$ on the unit hypersphere $S^{m}$, $u^{1}(p), \cdots, u^{m+1}(p)$ are the rectangular coordinates in $R^{m+1}$ of $p$. Then we have

$$
\left(u^{1}\right)^{2}+\cdots+\left(u^{m+1}\right)^{2}=1 .
$$

If we use $x^{1}, \cdots, x^{m}$ as the local coordinates of $S^{m}$ and if the local components of the standard Riemannian metric $g$ of $S^{m}$ are denoted by $g_{\mu \lambda}$, then we have

$$
g_{\mu \lambda}=\sum_{h} B_{\mu}^{h} B_{\lambda}^{h}, \quad B_{\lambda}^{h}=\partial u^{h} / \partial x^{\lambda} .
$$

As it is well-known, $\left\{u^{1}, \cdots, u^{m+1}\right\}$ is an orthonormal basis of the space of eigenfunctions of the Laplace-Beltrami operator $\Delta=-g^{\mu \lambda} \nabla_{\mu} \nabla_{\lambda}$ on $\left(S^{m}, g\right)$ satisfying

$$
\begin{gathered}
\Delta u^{n}=\lambda_{1} u^{n}, \quad \lambda_{1}=m, \\
\int_{S m} u^{j} u^{\imath} d \omega=\frac{1}{m+1} \delta^{j i} \int_{S m} d \omega
\end{gathered}
$$

where $d \omega$ is the volume element of $\left(S^{m}, g\right)$.

Let $V_{s}$ be the space of eigenfunctions $f$ of $\Delta$ satisfying

$$
\Delta f=\lambda_{s} f, \quad \lambda_{s}=s(s+m-1) .
$$

Then we put

$$
\begin{aligned}
n & =\operatorname{dim} V_{s} \\
& =(2 s+m-1)(s+m-2) ! /(s !(m-1) !)
\end{aligned}
$$

and take $n$ linearly independent eigenfunctions $f^{A} \in V_{s}(A=1, \cdots, n)_{\varepsilon}$ such that

$$
\Sigma_{A}\left(f^{A}\right)^{2}=r^{2}
$$

where $r$ is a positive constant. Taking an orthonormal basis $\left\{\tilde{e}_{1}, \cdots, \tilde{e}_{n}\right\}$ of $R^{n}$, we have an immersion $f: S^{m} \rightarrow S^{n-1}$ induced by $f^{A} \tilde{e}_{A}$, where $S^{n-1}$ is a hypersphere of radius $r$ in $R^{n}$. In the sequel this understanding is continued. But, in general, such an immersion may or may not be isometric and minimal. $f$ is 
an isometric minimal immersion if and only if $f$ satisfies besides (2.5) the condition

$$
\sum_{A} B_{\mu}^{A} B_{\lambda}^{A}=g_{\mu \lambda}, \quad B_{\lambda}^{A}=\partial f^{A} / \partial x^{\lambda} .
$$

We denote the linear space of covariant symmetric tensors of degree $s$ of $R^{m+1}$ by $\widetilde{S}_{s}$. If $t \in \widetilde{S}_{s}$ and $v_{1}, \cdots, v_{s}$ are vectors of $R^{m+1}$, then $t\left(v_{1}, \cdots, v_{s}\right) \in R$. We define a linear subspace $S_{s}$ of $\tilde{S}_{s}$ by saying that $t \in S_{s}$ if and only if $t \in \widetilde{S}_{s}$ and

$$
\sum_{i} t\left(e_{\imath}, e_{\imath}, v_{3}, \cdots, v_{s}\right)=0
$$

for any $s-2$ vectors $v_{3}, \cdots, v_{s}$ and an orthonormal basis $\left\{e_{1}, \cdots, e_{m+1}\right\}$ of $R^{m+1}$. In the present paper we always use $e_{2}$ in this sense. The following fact is well-known. $t(u, \cdots, u)$, where $t \in S_{s}$ and $u$ is the vector field on $S^{m}$ whose rectangular components in $R^{m+1}$ are $u^{1}, \cdots, u^{m+1}$ as we have stated above, is an eigenfunction of the Laplacian on $S^{m}$ satisfying

$$
\Delta t(u, \cdots, u)=\lambda_{s} t(u, \cdots, u) .
$$

Conversely, for any eigenfunction $f$ of $\Delta$ satisfying (2.3) there exists a tensor $t \in S_{s}$ satisfying $t(u, \cdots, u)=f$. This means that there exists a mapping $\varphi: V_{s} \rightarrow S_{s}$ with the inverse $\varphi^{-1}: S_{s} \rightarrow V_{s}$. It is to be understood that in the sequel we use the letter $u$ only in this sense.

Now we consider eigenfunctions $f^{A}$ satisfying (2.3), (2.5). The $n$ tensors $t^{A} \in S_{s}$ such that $t^{A}(u, \cdots, u)=f^{A}$ are then denoted by $F^{A}\left(=\varphi\left(f^{A}\right)\right)$. From these tensors $F^{A}$ we get a covariant tensor $f_{s, s}$ of $R^{m+1}$ of degree $2 s$ such that $f_{s, s}\left(v_{1}\right.$, $\left.\cdots, v_{s} ; v_{s+1}, \cdots, v_{2 s}\right)$ is symmetric both in $v_{1}, \cdots, v_{s}$ and in $v_{s+1}, \cdots, v_{2 s}$ and

by putting

$$
\begin{aligned}
& f_{s, s}\left(v_{1}, \cdots, v_{s} ; v_{s+1}, \cdots, v_{2 s}\right) \\
& \quad=f_{s, s}\left(v_{s+1}, \cdots, v_{2 s} ; v_{1}, \cdots, v_{s}\right)
\end{aligned}
$$

$$
\begin{aligned}
& f_{s, s}\left(v_{1}, \cdots, v_{s} ; v_{s+1}, \cdots, v_{2 s}\right) \\
& \quad=\Sigma_{A} F^{A}\left(v_{1}, \cdots, v_{s}\right) F^{A}\left(v_{s+1}, \cdots, v_{2 s}\right) .
\end{aligned}
$$

$f_{s, s}$ is called the tensor of degree $2 s$ associated with the immersion $f$. We can also consider a tensor $F$ of $R^{m+1}$ of degree $s$ and with value in $R^{n}$ associated with the immersion $f$ such that the components in $R^{n}$ are $F^{A}$.

In order to study the property of $f_{s, s}$, we define the space $\tilde{B}_{s, s}$ of covariant bi-symmetric tensors of $R^{m+1}$ of bi-degree $(s, s)$ by saying that $b_{s, s} \in \tilde{B}_{s s}$ if and only if $b_{s, s}$ is a covariant tensor of $R^{m+1}$ of degree $2 s$ satisfying 


$$
\begin{aligned}
& b_{s, s}\left(v_{1}, \cdots, v_{s} ; v_{s+1}, \cdots, v_{2 s}\right) \\
& \quad=b_{s, s}\left(v_{s+1}, \cdots, v_{2 s} ; v_{1}, \cdots, v_{s}\right) \\
& \quad=b_{s, s}\left(v_{P(1)}, \cdots, v_{P(s)} ; v_{s+1}, \cdots, v_{2 s}\right)
\end{aligned}
$$

for every permutation $P$ of $1, \cdots, s$ and every vectors $v_{1}, \cdots, v_{2 s}$ of $R^{m+1}$. A linear subspace $B_{s, s}$ of $\widetilde{B}_{s, s}$ is defined by saying $b_{s, s} \in B_{s, s}$ if and only if $b_{s, s} \in$ $\tilde{B}_{s, s}$ and satisfies

$$
\sum_{i} b_{s, s}\left(e_{\imath}, e_{\imath}, v_{3}, \cdots, v_{s} ; v_{s+1}, \cdots, v_{2 s}\right)=0 .
$$

As $t \in S_{s}$ satisfies (2.7), $f_{s, s}$ satisfies (2.11), namely, $f_{s, s} \in B_{s, s}$. We call $B_{s, s}$ the space of bi-symmetric harmonic tensors of bi-degree $(s, s)$.

An immersion $f: S^{m} \rightarrow S^{n-1}$ caused by eigenfunctions $f^{A}$ satisfying (2.3) and (2.5) is called an immersion of order $s$. Thus we have obtained the following lemma.

LEMma 2.1. There exists a mapping $\Phi$ of the space $\mathfrak{F}$ of equivalence classes of isometric minimal immersions of order $s$ into $B_{s, s}$.

Then there arises the following problems.

$(\alpha)$ What is the image of the equivalence class $\mathfrak{h}$ of the standard minimal immersions?

( $\beta$ ) How is the relation between the image of the equivalence class $\mathfrak{h}$ of the standard minimal immersions and the image of an equivalence class $\mathfrak{f}$ of non standard isometric minimal immersions?

$(\alpha)$ is answered in $\S 5$. ( $\beta)$ is studied in $\S 6$.

\section{Some properties of the tensor $f_{s, s}$ of an isometric minimal immersion.}

We denote the Riemannian connection on $\left(S^{m}, g\right)$ by $\nabla$. If $a$ is a function on $S^{m}$ we understand $\nabla_{\lambda} a$ to be the usual partial derivative $\partial_{\lambda} a$.

As we have explained in $\S 2$, we have, for each eigenfunction $f^{A}$, the tensor $F^{A}$ satisfying $f^{A}=F^{A}(u, \cdots, u)$. As $F^{A}$ is a symmetric tensor, we get

$$
\nabla_{\lambda} f^{A}=s F^{A}\left(\nabla_{\lambda} u\right)
$$

where $F^{A}\left(\nabla_{\lambda} u\right)$ stands for $F^{A}\left(\nabla_{\lambda} u, u, \cdots, u\right)$. Similarly we abbreviate $F^{A}(u, \cdots, u)$ to $F^{A}(u)$ or even to $F^{A}$, hence $f^{A}=F^{A}$, if there is no possibility of confusion, and $F^{A}\left(\nabla_{\lambda} u, \cdots, \nabla_{\kappa} u, u, \cdots, u\right)$ to $F^{A}\left(\nabla_{\lambda} u, \cdots, \nabla_{\kappa} u\right)$.

On the other hand we have

$$
\begin{gathered}
\nabla_{\mu} u^{i} \nabla^{\mu} u^{h}=\delta^{i h}-u^{2} u^{h}, \\
\nabla_{\mu} \nabla_{\lambda} u^{h}=-g_{\mu \lambda} u^{h}
\end{gathered}
$$


where $\nabla^{\mu}=g^{\mu \lambda} \nabla_{\lambda}$. As we use rectangular coordinates in $R^{m+1}$, we can freely write $u_{h}$ for $u^{h}$ so that (2.1) may be written $u_{i} u^{\imath}=1, \delta_{i h} u^{2} u^{h}=1$ or $\delta^{i n} u_{i} u_{h}=1$. Thus we have $u_{i} \nabla_{i} u^{\imath}=0$ and

$$
\nabla_{\mu} u_{\imath} \nabla_{\lambda} u^{2}=g_{\mu \lambda} .
$$

From (2.5), (2.6) and (3.1) we get

$$
\Sigma_{A} f^{A} \nabla_{\lambda} f^{A}=0, \quad \text { namely, } \quad \Sigma_{A} F^{A}(u) F^{A}\left(\nabla_{\lambda} u\right)=0,
$$

$$
s^{2} \sum_{A} F^{A}\left(\nabla_{\mu} u\right) F^{A}\left(\nabla_{\lambda} u\right)=g_{\mu \lambda}
$$

which can be written in terms of $f_{s, s}$ as

$$
\begin{gathered}
f_{s, s}\left(\nabla_{\lambda} u ; u\right)=0, \\
f_{s, s}\left(\nabla_{\mu} u ; \nabla_{\lambda} u\right)=s^{-2} g_{\mu \lambda,}
\end{gathered}
$$

where $f_{s, s}\left(\nabla_{\lambda} u ; u\right)$ or $f_{s, s}\left(\nabla_{\lambda} u\right)$ and $f_{s, s}\left(\nabla_{\mu} u ; \nabla_{\lambda} u\right)$ stand for $f_{s, s}\left(\nabla_{\lambda} u, u, \cdots, u\right.$; $u, \cdots, u)$ and $f_{s, s}\left(\nabla_{\mu} u, u, \cdots, u ; \nabla_{\lambda} u, u, \cdots, u\right)$ respectively.

We also get from (3.1)

$$
\nabla_{\mu} \nabla_{\lambda} f^{A}=-s g_{\mu \lambda} f^{A}+s(s-1) F^{A}\left(\nabla_{\mu} u, \nabla_{\lambda} u\right),
$$

where we have used (3.3). We have

$$
\Sigma_{A} f^{A} \nabla_{\mu} \nabla_{\lambda} f^{A}=-\sum_{A} \nabla_{\mu} f^{A} \nabla_{\lambda} f^{A}=-g_{\mu \lambda}
$$

from (3.5) and (2.6), and, on the other hand,

$$
g^{\mu \lambda} F^{A}\left(\nabla_{\mu} u, \nabla_{\lambda} u\right)=-f^{A}
$$

in view of (2.7) and (3.2). Then we can deduce from (3.7) and (3.8) that $-m=$ $-s m r^{2}-s(s-1) r^{2}$ which proves

$$
r^{2}=m /(s(s+m-1)) .
$$

(3.7) is the formula for the second fundamental form $H_{\mu \lambda}{ }^{4}$ of the image $\imath \circ f\left(S^{m}\right)$ in $R^{n}$, where $i$ is the embedding $i: S^{n-1} \rightarrow R^{n}$.

From (3.7) we get, if $s \geqq 3$,

$$
\begin{aligned}
\nabla_{\nu} \nabla_{\mu} \nabla_{\lambda} f^{A}= & -s g_{\mu \lambda} \nabla_{\nu} f^{A}-(s-1)\left(g_{\nu \mu} \nabla_{\lambda} f^{A}+g_{\nu \lambda} \nabla_{\mu} f^{A}\right) \\
& +s(s-1)(s-2) F^{A}\left(\nabla_{\nu} u, \nabla_{\mu} u, \nabla_{\lambda} u\right) .
\end{aligned}
$$

As we have

and

$$
\sum_{A} f^{A} \nabla_{\nu} \nabla_{\mu} \nabla_{\lambda} f^{A}=\nabla_{\nu}\left(\sum_{A} f^{A} \nabla_{\mu} \nabla_{\lambda} f^{A}\right)-\sum_{A} \nabla_{\nu} f^{A} \nabla_{\mu} \nabla_{\lambda} f^{A}
$$

$$
\sum_{A} \nabla_{\nu} f^{A} \nabla_{\mu} \nabla_{\lambda} f^{A}=\sum_{A} B_{\nu}^{A} H_{\mu}{ }^{A}=0,
$$


we get in view of (3.5) and (3.8)

$$
f_{s, s}\left(\nabla_{\nu} u, \nabla_{\mu} u, \nabla_{\lambda} u\right)=0
$$

if $s \geqq 3$. If $s=2$, we have the simple formula

$$
\nabla_{\nu} \nabla_{\mu} \nabla_{\lambda} f^{A}=-2 g_{\mu \lambda} \nabla_{\nu} f^{A}-g_{\nu \mu} \nabla_{\lambda} f^{A}-g_{\nu \lambda} \nabla_{\mu} f^{A}
$$

which implies that the second fundamental form is parallel in the normal bundle. From (3.11) we get, in view of (3.5) and (3.7),

$$
f_{s, s}\left(\nabla_{\nu} u ; \nabla_{\mu} u, \nabla_{\lambda} u\right)=0 .
$$

Now let us define a covariant tensor $\chi_{p}$ of degree $p$ by

$$
\chi_{p}\left(v_{1}, \cdots, v_{p}\right)=f_{s, s}\left(v_{1}, \cdots, v_{p}, u, \cdots, u ; u, \cdots, u\right)
$$

and a covariant tensor $\chi_{q, p}$ of degree $p+q$ by

$$
\begin{aligned}
& \chi_{q, p}\left(w_{1}, \cdots, w_{q} ; v_{1}, \cdots, v_{p}\right) \\
& \quad=f_{s, s}\left(w_{1}, \cdots, w_{q}, u, \cdots, u ; v_{1}, \cdots, v_{p}, u, \cdots, u\right) .
\end{aligned}
$$

These are in fact tensor valued functions on $S^{m}$ except the case $p=q=s$.

We have from $(3.5)^{\prime}$

$$
\chi_{1}\left(\nabla_{\lambda} u\right)=0
$$

From (3.7) and (3.8) we get

hence

$$
-g_{\mu \lambda}=-s g_{\mu \lambda} r^{2}+s(s-1) \chi_{2}\left(\nabla_{\mu} u, \nabla_{\lambda} u\right),
$$

$$
\chi_{2}\left(\nabla_{\mu} u, \nabla_{\lambda} u\right)=-(s(s+m-1))^{-1} g_{\mu \lambda} .
$$

$(3.6)^{\prime},(3.12)$ and (3.13) are equivalent respectively to

$$
\begin{aligned}
& \chi_{1,1}\left(\nabla_{\mu} u ; \nabla_{\lambda} u\right)=s^{-2} g_{\mu \lambda}, \\
& \chi_{3}\left(\nabla_{\nu} u, \nabla_{\mu} u, \nabla_{\lambda} u\right)=0, \\
& \chi_{1,2}\left(\nabla_{\nu} u ; \nabla_{\mu} u, \nabla_{\lambda} u\right)=0 .
\end{aligned}
$$

At any point of $S^{m}$ the $m+1$ vectors $u, \nabla_{1} u, \cdots, \nabla_{m} u$ are linearly independent vectors of $R^{m+1}$. In the sequel we denote by $\langle$,$\rangle the natural metric in R^{m+1}$ so that we have $\langle u, u\rangle=1,\left\langle u, \nabla_{\lambda} u\right\rangle=0$. Thus (3.16) implies $\chi_{1}(v-\langle v, u\rangle u)=0$ for an arbitrary vector $v$ of $R^{m+1}$. Consequently we get

$$
\begin{aligned}
\chi_{1}(v) & =\chi_{1}(v-\langle v, u\rangle u+\langle v, u\rangle u) \\
& =\langle v, u\rangle \chi_{1}(u)=\langle v, u\rangle \Sigma_{A} f^{A} f^{A}=r^{2}\langle v, u\rangle,
\end{aligned}
$$


namely

$$
\chi_{1}(v)=(m /(s(s+m-1)))\langle u, v\rangle .
$$

As for $\chi_{2}$ we have, in view of (3.4), $g_{\mu \lambda}=\left\langle\nabla_{\mu} u, \nabla_{\lambda} u\right\rangle, \chi_{2}\left(\nabla_{\mu} u, \nabla_{\lambda} u\right)=$ $-(s(s+m-1))^{-1}\left\langle\nabla_{\mu} u, \nabla_{\lambda} u\right\rangle$ and $\chi_{2}\left(u, \nabla_{\lambda} u\right)=\chi_{1}\left(\nabla_{\lambda} u\right)=0$. Thus we have

$$
\begin{aligned}
& \chi_{2}\left(v_{1}, v_{2}\right)=-(s(s+m-1))^{-1}\left\langle v_{1}, v_{2}\right\rangle, \\
& \chi_{2}\left(u, v_{1}\right)=\chi_{2}\left(u, v_{2}\right)=0
\end{aligned}
$$

if $\left\langle u, v_{1}\right\rangle=\left\langle u, v_{2}\right\rangle=0$. Consequently, for arbitrary vectors $v_{1}$ and $v_{2}$, we have, in view of $\chi_{2}(u, u)=\chi_{1}(u)=r^{2}$,

namely,

$$
\chi_{2}\left(v_{1}, v_{2}\right)=\chi_{2}\left(v_{1}-\left\langle v_{1}, u\right\rangle u+\left\langle v_{1}, u\right\rangle u, v_{2}-\left\langle v_{2}, u\right\rangle u+\left\langle v_{2}, u\right\rangle u\right)
$$$$
=-(s(s+m-1))^{-1}\left\langle v_{1}-\left\langle v_{1}, u\right\rangle u, v_{2}-\left\langle v_{2}, u\right\rangle u\right\rangle+\left\langle v_{1}, u\right\rangle\left\langle v_{2}, u\right\rangle r^{2} \text {, }
$$

$$
\chi_{2}\left(v_{1}, v_{2}\right)=\frac{m+1}{s(s+m-1)}\left\langle u, v_{1}\right\rangle\left\langle u, v_{2}\right\rangle-\frac{1}{s(s+m-1)}\left\langle v_{1}, v_{2}\right\rangle .
$$

In such a way we get

$$
\begin{aligned}
& \chi_{1,1}(w ; v)=\frac{(s-1)(m-1)}{s^{2}(s+m-1)}\langle u, w\rangle\langle u, v\rangle+\frac{1}{s^{2}}\langle w, v\rangle, \\
& \chi_{3}\left(v_{1}, v_{2}, v_{3}\right)=\frac{m+3}{s(s+m-1)}\left\langle u, v_{1}\right\rangle\left\langle u, v_{2}\right\rangle\left\langle u, v_{3}\right\rangle \\
& -\frac{1}{2 s(s+m-1)} \Sigma_{P}\left\langle u, v_{P(1)}\right\rangle\left\langle v_{P(2)}, v_{P(3)}\right\rangle, \\
& \chi_{1,2}\left(w ; v_{1}, v_{2}\right)=\frac{(s-2)(m-1)}{s^{2}(s+m-1)}\langle u, w\rangle\left\langle u, v_{1}\right\rangle\left\langle u, v_{2}\right\rangle \\
& -\frac{1}{s(s+m-1)}\langle u, w\rangle\left\langle v_{1}, v_{2}\right\rangle \\
& +\frac{1}{s^{2}}\left(\left\langle w, v_{1}\right\rangle\left\langle u, v_{2}\right\rangle+\left\langle w, v_{2}\right\rangle\left\langle u, v_{1}\right\rangle\right)
\end{aligned}
$$

where $v_{1}, v_{2}, v_{3}, v, w$ are arbitrary vectors of $R^{m+1}$.

As we have $\chi_{q, p}\left(w_{1}, \cdots, w_{q} ; v_{1}, \cdots, v_{p}\right)=\chi_{p, q}\left(v_{1}, \cdots, v_{p} ; w_{1}, \cdots, w_{q}\right), \chi_{q, p+1}\left(w_{1}\right.$, $\left.\cdots, w_{q} ; v_{1}, \cdots, v_{p}, u\right)=\chi_{q, p}\left(w_{1}, \cdots, w_{q} ; v_{1}, \cdots, v_{p}\right)$ and $\chi_{0, p}=\chi_{p, 0}=\chi_{p}$, the equations (3.21), (3.22), (3.23) are satisfied by (3.24), (3.25). Though these equations are useful, it is also important to notice that (3.22) is sufficient for an immersion $f$ of order $s$ to be isometric and minimal.

In order to prove this, suppose that $f^{A}$ are eigenfunctions satisfying (2.3) and (3.22). From (3.22) we get $\chi_{2}(u, u)=r^{2}$ which shows that (2.5) is satisfied. Hence we have, for an arbitrary vector $w$ of $R^{m+1}$, 


$$
f_{s, s}(w, \cdots, w ; w, \cdots, w)=\langle w, w\rangle^{s} r^{2} .
$$

From this equation we get, for arbitrary vectors $v, v_{1}, v_{2}, w$ of $R^{m+1}$,

$$
\begin{aligned}
& f_{s, s}(v, w, \cdots, w ; w, \cdots, w)=\langle v, w\rangle\langle w, w\rangle^{s-1} r^{2}, \\
& (s-1) f_{s, s}\left(v_{1}, v_{2}, w, \cdots, w ; w, \cdots, w\right) \\
& \quad+s f_{s, s}\left(v_{1}, w, \cdots, w ; v_{2}, w, \cdots, w\right) \\
& \quad=\left(\left\langle v_{1}, v_{2}\right\rangle\langle w, w\rangle^{s-1}+2(s-1)\left\langle v_{1}, w\right\rangle\left\langle v_{2}, w\right\rangle\langle w, w\rangle^{s-2}\right) r^{2},
\end{aligned}
$$

hence

$$
\begin{aligned}
& (s-1) \chi_{2}\left(v_{1}, v_{2}\right)+s \chi_{1,1}\left(v_{1} ; v_{2}\right) \\
& =\left(\left\langle v_{1}, v_{2}\right\rangle+2(s-1)\left\langle u, v_{1}\right\rangle\left\langle u, v_{2}\right\rangle\right) r^{2} .
\end{aligned}
$$

This proves that (3.23) is a result of (3.22). As we easily get (2.6) from (3.23), $f: S^{m} \rightarrow S^{n-1}$ is an isometric minimal immersion. Thus we have the following lemma.

LEMMA 3.1. Let $f: S^{m} \rightarrow S^{n-1}$ be an zmmersion of order $s$ such that $n$ and $r$ are the numbers given in $\S 1$. Then (3.22) is a necessary and sufficient condition for this immersion $f$ to be isometruc and minimal.

THEOREM 3.2. If $s=3$, the tensor $f_{s, s}$ of an isometric minumal immersion $f$ is a tensor such that

$$
\begin{aligned}
f_{3,3}\left(w_{1}, w_{2},\right. & \left.\left.w_{3} ; v_{1}, v_{2}, v_{3}\right)=\frac{m+3}{3 ! 3(m+2)}(*)-\frac{1}{3 ! 3(m+2)}{ }^{* *}\right), \\
(*)= & \Sigma_{P}\left\langle w_{P(1)}, v_{1}\right\rangle\left\langle w_{P(2)}, v_{2}\right\rangle\left\langle w_{P(3)}, v_{3}\right\rangle \\
(* *)= & \Sigma_{P}\left(\left\langle w_{P(1)}, v_{1}\right\rangle\left\langle w_{P(2)}, w_{P(3)}\right\rangle\left\langle v_{2}, v_{3}\right\rangle\right. \\
& +\left\langle w_{P(1)}, v_{2}\right\rangle\left\langle w_{P(2)}, w_{P(3)}\right\rangle\left\langle v_{3}, v_{1}\right\rangle \\
& \left.+\left\langle w_{P(1)}, v_{3}\right\rangle\left\langle w_{P(2)}, w_{P(3)}\right\rangle\left\langle v_{1}, v_{2}\right\rangle\right) .
\end{aligned}
$$

Proof. When $s=3$, (3.24) is equivalent to

$$
\begin{gathered}
f_{3,3}\left(v_{1}, v_{2}, v_{3} ; u, u, u\right)-\frac{m+3}{3(m+2)}\left\langle u, v_{1}\right\rangle\left\langle u, v_{2}\right\rangle\left\langle u, v_{3}\right\rangle \\
+\frac{1}{3(m+2)}\langle u, u\rangle\left(\left\langle u, v_{1}\right\rangle\left\langle v_{2}, v_{3}\right\rangle+\left\langle u, v_{2}\right\rangle\left\langle v_{3}, v_{1}\right\rangle\right. \\
\left.+\left\langle u, v_{3}\right\rangle\left\langle v_{1}, v_{2}\right\rangle\right)=0
\end{gathered}
$$

The left-hand-side can be written in the form $c_{k j i} u^{k} u^{j} u^{2}$ with constant coefficients $c_{k j i}$ which are symmetric in $k, j, i$. Hence $c_{k j i}=0$ and we get $c_{k j i} w_{1}^{k} w_{2}^{j} w_{3}^{2}=0$. This proves $(3.26)_{3}$. 
Now let us notice that, according to the definition, the tensor $f_{s, s}$ induces a mapping $\varphi_{s, s}: S^{m} \times S^{m} \rightarrow R$ such that

$$
\varphi_{s, s}(p, q)=\Sigma_{A} f^{A}(p) f^{A}(q)
$$

where $f^{A}(p)=F^{A}(u(p), \cdots, u(p))$. If $\left\{f^{1}, \cdots, f^{n}\right\}$ is an orthonormal basis of the eigenspace $V_{s}$, then the set $\left\{f^{1}, \cdots, f^{n}\right\}$ induces an isometric minimal immersion $f: S^{m} \rightarrow S^{n-1}$ [1]. If $\left\{f^{1}, \cdots,{ }^{\prime} f^{n}\right\}$ is also a set of eigenfunctions in $V_{s}$, then we have

$$
{ }^{\prime} f^{A}=S_{B}{ }^{A} f^{B}
$$

where $\left[S_{B}{ }^{A}\right]$ is a constant matrix on $S^{m}$. If, moreover, $\left\{f^{1}, \cdots, f^{n}\right\}$ induces an isometric minimal immersion ' $f: S^{m} \rightarrow S^{n-1}$ such that the tensor ' $f_{s, s}$ associated with ' $f$ is equal to the tensor $f_{s, s}$ associated with $f$, then we have

namely,

$$
\Sigma_{A} f^{A}(p)^{\prime} f^{A}(q)=\Sigma_{A} f^{A}(p) f^{A}(q),
$$

$$
\Sigma_{A} S_{C}{ }^{A} S_{B}{ }^{A} f^{C}(p) f^{B}(q)=\Sigma_{A} f^{A}(p) f^{A}(q)
$$

As $q$ can move freely on $S^{m}$ independent of $p$, we get

$$
\Sigma_{A} S_{C}{ }^{A} S_{B}{ }^{A} f^{C}(p)=f^{B}(p) .
$$

As $p$ can move freely on $S^{m}$, we get

$$
\sum_{A} S_{C}{ }^{A} S_{B}{ }^{A}=\delta_{C B},
$$

hence $\left[S_{B}{ }^{A}\right]$ is an orthogonal matrix and the immersion ' $f$ is equivalent to the immersion $f$. It is easy to see that a similar result is obtained when $\left\{f^{1}, \cdots, f^{n}\right\}$ is any basis of $V_{s}$ inducing an isometric minimal immersion $f: S^{m} \rightarrow S^{n-1}$. Thus we have the following theorem.

Theorem 3.3. If $f: S^{m} \rightarrow S^{n-1}$ and ' $f: S^{m} \rightarrow S^{n-1}$ are full isometrac minmmal immersions of order $s$, such that $f_{s, s}=^{\prime} f_{s, s}$, then ' $f$ is equivalent to $f$.

If $s=3, f_{s, s}$ is given by $(3.26)_{3}$. Thus Theorem 3.2 and Theorem 3.3 prove the rigidity property of isometric minimal immersions of order 3 [1].

\section{The unit element of $B_{s, s}$.}

In the foregoing paragraph we found that, if $f$ is an isometric minimal immersion: $S^{m} \rightarrow S^{n-1}$ of order 3 , then the tensor $f_{3,3}$ is given by $(3.26)_{3}$. If the order is $2, f_{2,2}$ is easily obtained from (3.22) in the form 


$$
\begin{aligned}
f_{2,2}\left(w_{1}, w_{2} ; v_{1}, v_{2}\right)= & \frac{1}{4}\left(\left\langle w_{1}, v_{1}\right\rangle\left\langle w_{2}, v_{2}\right\rangle+\left\langle w_{2}, v_{1}\right\rangle\left\langle w_{1}, v_{2}\right\rangle\right) \\
& -\frac{1}{2(m+1)}\left\langle w_{1}, w_{2}\right\rangle\left\langle v_{1}, v_{2}\right\rangle
\end{aligned}
$$

which is just the formula (7.14) of [6].

If $s \geqq 4, f_{s, s}$ acquires some freedom which increases rapidly with $s$. In order to study this phenomenon we first define the unit element $U$ of $B_{s, s}$.

As $R^{m+1}$ is endowed with the natural Riemannian metric $\langle$,$\rangle , we can extend$ the inner product to $S_{s}$ by the formula

$$
\left\langle t_{1}, t_{2}\right\rangle=\sum_{\imath_{1} \cdots \imath_{s}} t_{1}\left(e_{\imath_{1}}, \cdots, e_{\imath_{s}}\right) t_{2}\left(e_{\imath_{1}}, \cdots, e_{\imath_{s}}\right) .
$$

Similarly, if $b$ is an element of $B_{s, s}$ and $t$ an element of $S_{s}$, we can define the action of $B_{s, s}$ on $S_{s}, b \circ t$ and $t \circ b$, by

$$
\begin{aligned}
(b \circ t) & \left(v_{1}, \cdots, v_{s}\right)=(t \circ b)\left(v_{1}, \cdots, v_{s}\right) \\
& =\Sigma_{\imath_{1} \cdots \imath_{s}} b\left(v_{1}, \cdots, v_{s} ; e_{\imath_{1}}, \cdots, e_{\imath_{s}}\right) t\left(e_{\imath_{1}}, \cdots, e_{\imath_{s}}\right) \\
& =\Sigma_{\imath_{1} \cdots \imath_{s}} t\left(e_{\imath_{1}}, \cdots, e_{\imath_{s}}\right) b\left(e_{\imath_{1}}, \cdots, e_{\imath_{s}} ; v_{1}, \cdots, v_{s}\right) .
\end{aligned}
$$

This implies that $b$ acts as a two-sided linear operator on $S_{s}$. The unit element $U$ of $B_{s, s}$ is defined by

$$
U \circ t=t
$$

where $t$ is any element of $S_{s}$.

We try to put

$$
\begin{aligned}
& U\left(w_{1}, \cdots, w_{s} ; v_{1}, \cdots, v_{s}\right)=a_{0} \widetilde{S}_{(w)}\left\langle w_{1}, v_{1}\right\rangle \cdots\left\langle w_{s}, v_{s}\right\rangle \\
& +a_{1} \mathfrak{S}_{(w)} \mathfrak{S}_{(v)}\left\langle w_{1}, w_{2}\right\rangle\left\langle v_{1}, v_{2}\right\rangle\left\langle w_{3}, v_{3}\right\rangle \cdots\left\langle w_{s}, v_{s}\right\rangle \\
& +\cdots \cdots \cdots . . . \\
& +a_{p} \mathfrak{S}_{(w)} \mathfrak{S}_{(v)}\left\langle w_{1}, w_{2}\right\rangle \cdots\left\langle w_{2 p-1}, w_{2 p}\right\rangle \\
& \left\langle v_{1}, v_{2}\right\rangle \cdots\left\langle v_{2 p-1}, v_{2 p}\right\rangle \\
& \left\langle w_{2 p+1}, v_{2 p+1}\right\rangle \cdots\left\langle w_{s}, v_{s}\right\rangle \text {, }
\end{aligned}
$$

where $\mathfrak{S}_{(w)}$ (resp. $\left.\mathfrak{S}_{(v)}\right)$ means the symmetric part with respect to $w_{1}, \cdots, w_{s}$ (resp. $v_{1}, \cdots, v_{s}$ ) and $a_{p} \neq 0$. The number $p, p \leqq[s / 2]$, and the coefficients $a_{0}, a_{1}, \cdots, a_{p}$ are chosen in such a way that $U$ satisfies

$$
\sum_{i} U\left(e_{\imath}, e_{\imath}, v_{3}, \cdots, v_{s} ; v_{s+1}, \cdots, v_{2 s}\right)=0
$$

and (4.2). 
To that end, we first define $g_{r, s, s}\left(w_{1}, \cdots, w_{s} ; v_{1}, \cdots, v_{s}\right), 0 \leqq r \leqq p$, and $g_{r, s-2, s}\left(w_{3}, \cdots, w_{s} ; v_{1}, \cdots, v_{s}\right), 1 \leqq r \leqq p \leqq[s / 2]$, using $P, Q$ for permutations, by

$$
\begin{aligned}
& g_{r, s, s}\left(w_{1}, \cdots, w_{s} ; v_{1}, \cdots, v_{s}\right) \\
&=\sum_{P, Q}\left\langle w_{P(1)}, w_{P(2)}\right\rangle \cdots\left\langle w_{P(2 r-1)}, w_{P(2 r)}\right\rangle \\
&\left\langle v_{Q(1)}, v_{Q(2)}\right\rangle \cdots\left\langle v_{Q(2 r-1)}, v_{Q(2 r)}\right\rangle \\
&\left\langle w_{P(2 r+1)}, v_{Q(2 r+1)}\right\rangle \cdots\left\langle w_{P(s)}, v_{Q(s)}\right\rangle, \\
& g_{r, s-2, s}\left(w_{3}, \cdots, w_{s} ; v_{1}, \cdots, v_{s}\right) \\
&=\sum_{P, Q}\left\langle w_{P(3)}, w_{P(4)}\right\rangle \cdots\left\langle w_{P(2 r-1)}, w_{P(2 r)}\right\rangle \\
&\left\langle v_{Q(1)}, v_{Q(2)}\right\rangle \cdots\left\langle v_{Q(2 r-1)}, v_{Q(2 r)}\right\rangle \\
&\left\langle w_{P(2 r+1)}, v_{Q(2 r+1)}\right\rangle \cdots\left\langle w_{P(s)}, v_{Q(s)}\right\rangle
\end{aligned}
$$

where $w_{1}, \cdots, w_{s}, v_{1}, \cdots, v_{s}$ are arbitrary vectors of $R^{m+1}$ again and $P$ in (4.6) is a permutation of $3, \cdots, s$.

Then we get

$$
\begin{aligned}
& \sum_{i} g_{r, s, s}\left(e_{\imath}, e_{\imath}, w_{3}, \cdots, w_{s} ; v_{1}, \cdots, v_{s}\right) \\
&= 2 r(2 s-2 r+m-1) g_{r, s-2, s}\left(w_{3}, \cdots, w_{s} ; v_{1}, \cdots, v_{s}\right) \\
&+(s-2 r)(s-2 r-1) g_{r+1, s-2, s}\left(w_{3}, \cdots, w_{s} ; v_{1}, \cdots, v_{s}\right)
\end{aligned}
$$

where the second term in the right-hand-side should be deleted if $2 r+2>s$. As (4.3) is equivalent to

$$
(s !)^{2} U=a_{0} g_{0, s, s}+a_{1} g_{1, s, s}+\cdots+a_{p} g_{p, s, s},
$$

we get, in view of (4.7),

$$
\begin{aligned}
& \left\{s(s-1) a_{0}+2(2 s+m-3) a_{1}\right\} g_{1} \\
& \quad+\left\{(s-2)(s-3) a_{1}+4(2 s+m-5) a_{2}\right\} g_{2} \\
& \quad+\cdots \cdots \cdots \cdots \\
& \quad+\left\{(s-2 p+2)(s-2 p+1) a_{p-1}+2 p(2 s+m-2 p-1) a_{p}\right\} g_{p} \\
& \quad+(s-2 p)(s-2 p-1) a_{p} g_{p+1} \\
& =0
\end{aligned}
$$

where $g_{r}$ stands for $g_{r, s-2, s}$.

From (4.6) we see that $g_{1}, \cdots, g_{r}$ are linearly independent if $2 r \leqq s$. Then we find from (4.9) that $s=2 p+1$ if $s$ is an odd number, and that $s=2 p$ if $s$ is 
an even number. The ratio of $a_{0}, a_{1}, \cdots, a_{p}$ is obtained from the system of equations

$$
(s-2 r+2)(s-2 r+1) a_{r-1}+2 r(2 s+m-2 r-1) a_{r}=0
$$

where $r$ ranges $1,2, \cdots, p$.

We can write (4.10) in the following form:

$$
\begin{aligned}
& \left(s^{2}-s\right) a_{0}+(2 m+4 s-6) a_{1}=0, \\
& \quad \cdots \ldots \ldots \ldots \ldots . . . \\
& \left\{\left(s^{2}-s\right)-4(r-1) s+2(r-1)(2 r-1)\right\} a_{r-1}+\{2 r m+4 r s-2 r(2 r+1)\} a_{r}=0, \\
& \quad \ldots \ldots \ldots \ldots \ldots \\
& \left\{\left(s^{2}-s\right)-4(p-1) s+2(p-1)(2 p-1)\right\} a_{p-1}+\{2 p m+4 p s-2 p(2 p+1)\} a_{p}=0 .
\end{aligned}
$$

Summing up these equations side by side we get

which becomes

$$
\begin{aligned}
& \left(s^{2}-s\right)\left(a_{0}+a_{1}+\cdots+a_{p-1}\right) \\
& \quad+2 m\left(a_{1}+2 a_{2}+\cdots+p a_{p}\right)+2 p(2 s-2 p-1) a_{p}=0
\end{aligned}
$$

$$
\left(s^{2}-s\right)\left(a_{0}+a_{1}+\cdots+a_{p}\right)+2 m\left(a_{1}+2 a_{2}+\cdots+p a_{p}\right)=0
$$

because of

$$
-s^{2}+s+2 p(2 s-2 p-1)=-(s-2 p)(s-2 p-1)=0 .
$$

If $t$ is any element of $S_{s}$, then we have

$$
\begin{gathered}
\sum_{\imath_{1} \cdots \ell_{s}} g_{r, s, s}\left(v_{1}, \cdots, v_{s} ; e_{\imath_{1}}, \cdots, e_{\imath_{s}}\right) t\left(e_{\imath_{1}}, \cdots, e_{\imath_{s}}\right) \\
=0 \quad \text { if } r>0 \\
=(s !)^{2} t\left(v_{1}, \cdots, v_{s}\right) \quad \text { if } r=0
\end{gathered}
$$

because of (2.7) and (4.5). Hence $U$ is the unit element of $B_{s, s}$ if we put $a_{0}=1$. Thus we have proved the following theorem.

THEOREM 4.1. The unit element $U$ of $B_{s, s}$ is given by (4.3) when $p$ and $a_{0}, a_{1}, \cdots, a_{p}$ are determined by

and (4.10).

$$
(s-2 p)(s-2 p-1)=0, \quad a_{0}=1
$$

\section{Standard minimal immersions.}

An isometric minimal immersion $f: S^{m} \rightarrow S^{n-1}$ is called a standard minimal immersion if the set of eigenfunctions $\left\{f^{1}, \cdots, f^{n}\right\}$ is an orthonormal basis of 
$V_{s}$, namely,

$$
\int_{M} f^{B} f^{A} d \omega=c \delta^{B A}
$$

where $M$ stands for $S^{m}, d \omega$ is the volume element of $S^{m}$ and $c$ is a certain constant.

LEMMA 5.1. (5.1) is equivalent to

$$
\left\langle F^{B}, F^{A}\right\rangle=c^{\prime} \delta^{B A},
$$

namely,

$$
\sum_{\imath_{1} \cdots \imath_{s}} F^{B}\left(e_{\imath_{1}}, \cdots, e_{\imath_{s}}\right) F^{A}\left(e_{\imath_{1}}, \cdots, e_{\imath_{s}}\right)=c^{\prime} \hat{o}^{B A}
$$

where $c^{\prime}$ is a constant and

$$
F^{A}(u, \cdots, u)=f^{A}
$$

Proof. When rectangular coordinates are taken in $R^{m+1}$ and the components of $F^{A}$ and $u$ are written $F_{\imath_{1} \ldots \varepsilon_{s}}{ }^{A}$ and $u^{2}$ respectively, (5.3) is written

$$
F_{\imath_{1} \cdots u_{s}}{ }^{A} u^{\imath_{1}} \cdots u^{\imath_{s}}=f^{A} .
$$

Now, for any natural number $q$ and any vector $v$ of $R^{m+1}$ we have, in view of the symmetry property of $S^{m}$,

$$
\int_{M}\langle u, v\rangle^{2 q} d \omega=k_{q}\langle v, v\rangle^{q}
$$

and especially

$$
\int_{M}\left(u^{1}\right)^{2 q} d \omega=k_{q}
$$

which determines $k_{q}$. As we have

and

$$
\delta_{i_{1} \imath_{2}} \cdots \delta_{i_{2 q-1} \imath_{2 q}} v^{\imath_{1}} \cdots v^{2_{2 q}}=\langle v, v\rangle^{q}
$$

we get

$$
\begin{aligned}
\langle u, v\rangle^{2 q} & =\delta_{\jmath_{1} 1_{1}} u^{\jmath_{1}} v^{\imath_{1}} \cdots \delta_{\jmath_{2 q} \imath_{2 q}} u^{\jmath_{2 q} v^{\imath_{2 q}}} \\
& =u_{\imath_{1}} \cdots u_{\imath_{2 q}} v^{\imath_{1}} \cdots v^{\imath_{2 q}}
\end{aligned}
$$

$$
\int_{M} u^{\imath_{1} \cdots} u^{\imath_{2 q}} d \omega=k_{q} \delta_{i_{1} \cdots \imath_{2 q}}=k_{q} \delta^{\imath_{1} \cdots \imath_{2 q}}
$$

where $\delta_{i_{1} \cdots l_{2 q}}$ is the symmetric part of $\delta_{i_{1} 2_{2}} \cdots \delta_{i_{2 q-1} \imath_{2 q}}$, namely,

$$
\delta_{i_{1} \cdots i_{2 q} q}=\mathbb{S}_{(i)} \delta_{i_{1} l_{2}} \cdots \delta_{i_{2 q-1{ }^{2} 2 q}} .
$$


Hence we have

$$
\begin{aligned}
& \int_{M} f^{B} f^{A} d \omega=F_{\imath_{1} \cdots \imath_{s}}^{B} F_{\imath_{s+1} \cdots \imath_{2 s}}{ }^{A} \int_{M} u^{\imath_{1}} \cdots u^{\imath_{2 s}} d \omega \\
& =k_{s} F_{\imath_{1} \cdots \imath_{s}}^{B} F_{\imath_{s+1} \cdots \imath_{2 s}}^{A} \delta^{\imath_{1} \cdots \imath_{2 s}} .
\end{aligned}
$$

$f^{A}$ being eigenfunctions, $F^{A}$ satisfy

and consequently we get

$$
\sum_{i} F_{i i_{3} \cdots i_{s}}^{A}=0
$$

$$
\begin{aligned}
& F_{\imath_{1} \cdots \imath_{s}}{ }^{B} F_{\imath_{s}+1 \cdots \imath_{2 s}}{ }^{A}{ }^{{ }^{2}{ }^{2} \cdots \imath_{2 s}} \\
& \quad=\frac{2^{s}(s !)^{2}}{(2 s) !} \delta^{\jmath_{1} \imath_{1}} \cdots \delta^{\jmath_{s} \imath_{s}} F_{{\jmath_{1} \cdots \jmath_{s}}^{B}}{ }^{B} F_{\imath_{1} \cdots \imath_{s}}{ }^{A}
\end{aligned}
$$

which proves the lemma.

At the same time we get

$$
(2 s) ! c=2^{s}(s !)^{2} k_{s} c^{\prime} .
$$

$c$ is obtained from

$$
n c=\int_{M} \Sigma_{A}\left(f^{A}\right)^{2} d \omega=r^{2} c_{m}
$$

where $c_{m}$ is the volume of the unit sphere $S^{m}$.

Remark. We easily get

$$
k_{q}=\frac{(m-1) ! !(2 q-1) ! !}{(2 q+m-1) ! !} c_{m} .
$$

From (4.3) we get

$$
U(u, \cdots, u ; u, \cdots, u)=a
$$

where $a=a_{0}+a_{1}+\cdots+a_{p}$ and $a_{0}=1$. Then the tensor $h_{s, s}$ defined by

$$
h_{s, s}=a^{-1} r^{2} U
$$

satisfies

$$
\begin{gathered}
h_{s, s}(u, \cdots, u ; u, \cdots, u)=r^{2}, \\
\int_{M} h_{s, s}(u, \cdots, u ; u, \cdots, u) d \omega=r^{2} c_{m} .
\end{gathered}
$$

Now, the inner product defined by (4.1) in $S_{s}$ can be naturally extended to the inner product in $\widetilde{S}_{s}$. If $C$ and $D$ belong to $\widetilde{S}_{s},\langle C, D\rangle$ is defined by

$$
\langle C, D\rangle=\sum_{\imath_{1} \cdots \imath_{s}} C\left(e_{\imath_{1}}, \cdots, e_{\imath_{s}}\right) D\left(e_{\imath_{1}}, \cdots, e_{\imath_{s}}\right) \text {. }
$$


We can also consider any element $A$ of $\tilde{B}_{s, s}$ as a two-sided linear operator acting on $\widetilde{S}_{s}$ according to the formula

$$
\begin{aligned}
(A \circ C) & \left(v_{1}, \cdots, v_{s}\right)=(C \circ A)\left(v_{1}, \cdots, v_{s}\right) \\
& =\sum_{\imath_{1} \cdots \imath_{s}} A\left(v_{1}, \cdots, v_{s} ; e_{\imath_{1}}, \cdots, e_{\imath_{s}}\right) C\left(e_{\imath_{1}}, \cdots, e_{\imath_{s}}\right) \\
& =\sum_{\imath_{1} \cdots l_{s}} C\left(e_{\imath_{1}}, \cdots, e_{\imath_{s}}\right) A\left(e_{\imath_{1}}, \cdots, e_{\imath_{s}} ; v_{1}, \cdots, v_{s}\right) .
\end{aligned}
$$

We denote by $P_{s}$ the orthogonal complement of $S_{s}$ in $\tilde{S}_{s}$. If we put $n^{\prime}=$ $\operatorname{dim} P_{s}$, we have

$$
n^{\prime}=\operatorname{dim} \widetilde{S}_{s}-\operatorname{dim} S_{s}=(s+m-2) ! /(m !(s-2) !) .
$$

We have the following theorem.

THEOREM 5.2. U is the projection operator: $\widetilde{S}_{s} \rightarrow S_{s}$.

Proof. Let $t_{\jmath_{1} \ldots \jmath_{s}} \in \widetilde{S}_{s}$ be the tensors defined by

$$
\begin{aligned}
t_{\jmath_{1} \cdots J_{s}}\left(v_{1}, \cdots, v_{s}\right) & =U\left(e_{\jmath_{1}}, \cdots, e_{\jmath_{s}} ; v_{1}, \cdots, v_{s}\right) \\
& =U\left(v_{1}, \cdots, v_{s} ; e_{\jmath_{1}}, \cdots, e_{\jmath_{s}}\right) .
\end{aligned}
$$

Then $t_{\jmath_{1} \cdots \jmath_{s}} \in S_{s}$, and every $b \in P_{s}$ satisfies

hence

$$
\sum_{\imath_{1} \cdots l_{s}} b\left(e_{\imath_{1}}, \cdots, e_{\imath_{s}}\right) t_{\partial_{1} \cdots \jmath_{s}}\left(e_{\imath_{1}}, \cdots, e_{\imath_{s}}\right)=0
$$

$$
\sum_{\imath_{1} \cdots \imath_{s}} b\left(e_{\imath_{1}}, \cdots, e_{\imath_{s}}\right) U\left(e_{\jmath_{1}}, \cdots, e_{\jmath_{s}} ; e_{\imath_{1}}, \cdots, e_{\imath_{s}}\right)=0 \text {. }
$$

On the other hand, we can put, in view of (4.3),

$$
\begin{aligned}
& U\left(e_{\jmath_{1}}, \cdots, e_{\jmath_{s}} ; e_{\imath_{1}}, \cdots, e_{\imath_{s}}\right) \\
& =\widetilde{S}_{(j)} \delta_{\jmath_{1} \imath_{1}} \cdots \delta_{\jmath_{s} \imath_{s}}+\Im_{(\jmath)} \delta_{\jmath_{1} \jmath_{2}} U^{*}\left(e_{\jmath_{3}}, \cdots, e_{\jmath_{s}}, e_{\imath_{1}}, \cdots, e_{\imath_{s}}\right)
\end{aligned}
$$

where $U^{*}$ is a certain tensor of degree $2 s-2$. As the tensor $E_{s, s} \in \tilde{B}_{s, s}$ defined by

$$
E_{s, s}\left(e_{\jmath_{1}}, \cdots, e_{\jmath_{s}} ; e_{\imath_{1}}, \cdots, e_{\imath_{s}}\right)=\Im_{(j)} \delta_{\jmath_{1} \imath_{1}} \cdots \delta_{\jmath_{s} \imath_{s}}
$$

is clearly the unit element of $\tilde{B}_{s, s}$ and $U$ satisfies $U \cdot U=U \cdot E_{s, s}=U$ as a linear operator by virtue of (4.4), we can see that $U$ and $V=E_{s, s}-U$ are projection operators such that $U: \widetilde{S}_{s} \rightarrow S_{s}, V: \widetilde{S}_{s} \rightarrow P_{s}$.

CoROLlary 5.3. An element $b$ of $\tilde{S}_{s}$ belongs to $P_{s}$ if and only if there exists an element $c$ of $\widetilde{S}_{s-2}$ such that

$$
b\left(v_{1}, \cdots, v_{s}\right)=\Im_{(v)}\left\langle v_{1}, v_{2}\right\rangle c\left(v_{3}, \cdots, v_{s}\right) .
$$


Proof. It is easy to see that, if $b$ satisfies (5.15), then $b$ belongs to $P_{s}$. Conversely, if $b$ belongs to $P_{s}$, we have $b=\left(E_{s, s}-U\right) b$. On the other hand, we get, from (5.13) and (5.14), $-\left(\left(E_{s, s}-U\right) b\right)\left(v_{1}, \cdots, v_{s}\right)=\mathfrak{S}_{(v)}\left\langle v_{1}, v_{2}\right\rangle c\left(v_{3}, \cdots, v_{s}\right)$ by choosing a suitable tensor $c$.

Let $\left\{a^{1}, \cdots, a^{n}\right\}$ and $\left\{a^{n+1}, \cdots, a^{n+n^{\prime}}\right\}$ be orthonormal bases of $S_{s}$ and $P_{s}$ respectively and let the indices $\xi, \eta, \zeta$ run the range $n+1, \cdots, n+n^{\prime}$. Then for any element $X$ of $\tilde{B}_{s, s}$ we have

$$
X=x_{B A} a^{B} \otimes a^{A}+x_{B \xi} a^{B} \otimes a^{\xi}+x_{\eta A} a^{\eta} \otimes a^{A}+x_{\eta \xi} a^{\eta} \otimes a^{\xi}
$$

where $x_{B A}=x_{A B}, x_{B \xi}=x_{\xi B}, x_{\eta \xi}=x_{\xi \eta}$. Especially, if $X$ belongs to $B_{s, s}$, we have $X=x_{B A} a^{B} \otimes a^{A}$.

If $f$ is a standard minimal immersion with eigenfunctions $f^{1}, \cdots, f^{n}$ and $F^{A}=\varphi\left(f^{A}\right)$, then $\left\{F^{1}, \cdots, F^{n}\right\}$ is an orthonormal basis of $S_{s}$ and satisfies (5.2) as we have pointed out in Lemma 5.1. Hence we can put

$$
U=u_{B A} F^{B} \otimes F^{A}
$$

where the coefficients $u_{B A}$ are obtained as follows. As $U$ is the unit element of $B_{s, s}$, we have, in view of $(4.2)$,

$$
u_{B A} F^{B}\left\langle F^{A}, F^{C}\right\rangle=\left(u_{B A} F^{B} \otimes F^{A}\right) \cdot F^{C}=F^{C},
$$

hence $c^{\prime} u_{B C} F^{B}=F^{C}$. Thus we get $u_{B A}=\delta_{B A} / c^{\prime}$, and consequently

$$
U=\frac{1}{c^{\prime}} \Sigma_{A} F^{A} \otimes F^{A} .
$$

From this result and (5.10) we get

$$
h_{s, s}=\frac{r^{2}}{a c^{\prime}} \sum_{A} F^{A} \otimes F^{A} .
$$

On the other hand, we have, from (5.17),

$$
U(u, \cdots, u ; u, \cdots u)=\frac{1}{c^{\prime}} \Sigma_{A}\left(f^{A}\right)^{2}=\frac{r^{2}}{c^{\prime}} .
$$

From this and (5.9) we get $a c^{\prime}=r^{2}$ and consequently

$$
h_{s, s}=\Sigma_{A} F^{A} \otimes F^{A},
$$

which proves that $h_{s, s}$ is the tensor of degree $2 s$ associated with the standard minimal immersion.

Thus we have the following theorem.

THEOREM 5.4. The unit element $U$ of $B_{s, s}$ and the tensors $F^{A}$ of a standard monmal immersion $f: S^{m} \rightarrow S^{n-1}$ are related by the equation 


$$
U=\frac{1}{c^{\prime}} \Sigma_{A} F^{A} \otimes F^{A}, \quad c^{\prime}=a^{-1} r^{2} .
$$

\section{Non standard isometric minimal immersions.}

In order to distinguish a standard minimal immersion from a general immersion, both of order $s$, we denote the former by $h$ and the latter by $f$ hereafter. The associated tensors of degree $s$ are denoted by $H^{A}$ and $F^{A}$ respectively and the associated tensors of degree $2 s$ are denoted by $h_{s, s}$ and $f_{s, s}$ respectively. Thus we can write

$$
h_{s, s}=\Sigma_{A} H^{A} \otimes H^{A}
$$

and

$$
U=\frac{1}{c^{\prime}} \Sigma_{A} H^{A} \otimes H^{A}
$$

Now let us take an arbitrary tensor $b_{s, s} \in B_{s, s}$ and look for the condition that there exists an immersion $f: S^{m} \rightarrow S^{n-1}$ of order $s$ such that $b_{s, s}=\sum_{A} F^{A} \otimes F^{A}$.

As $b_{s, s}$ is an element of $B_{s, s}$ and $\left\{H^{1}, \cdots, H^{n}\right\}$ is an orthonormal basis of $S_{s}$, we can express $b_{s, s}$ in the form

$$
b_{s, s}=b_{B A} H^{B} \otimes H^{A}
$$

where $b_{B A}=b_{A B}$. From this formula we immediately obtain the following lemma.

LEMMA 6.1. Let $b_{s, s}$ be an element of $B_{s, s}$. Then there exists a symmetric tensor of $R^{n}$ with components $b_{B A}$ such that (6.3) holds. A necessary and sufficient condition that there exists an immersion $f$ of order $s$ such that $b_{s, s}=\sum_{A} F^{A} \otimes F^{A}$ is that $b_{s, s}(u, \cdots, u ; u, \cdots, u)=r^{2}$ and $b_{B A}$ is non-negative. $f$ is full if and only if $\operatorname{rank}\left[b_{B A}\right]=n$.

Immersions considered in Lemma 6.1 are not in general isometric minimal immersions. We now study $f_{s, s}$ for an isometric minimal immersion. According to Lemma 3.1, an immersion $f: S^{m} \rightarrow S^{n-1}$ of order $s$ is isometric and minimal if and only if $f_{s, s}=\Sigma_{A} F^{A} \otimes F^{A}$ satisfies (3.22), hence

$$
\begin{aligned}
f_{s, s}\left(v_{1}, v_{2}, u, \cdots, u ; u, \cdots, u\right)= & \frac{m+1}{s(s+m-1)}\left\langle u, v_{1}\right\rangle\left\langle u, v_{2}\right\rangle \\
& -\frac{1}{s(s+m-1)}\left\langle v_{1}, v_{2}\right\rangle .
\end{aligned}
$$

When $f$ is an isometric minimal immersion, we put

$$
f_{s, s}=h_{s, s}+d_{s, s}
$$


for the associated tensor $f_{s, s}$. Then we have

$$
\begin{gathered}
d_{s, s}(u, \cdots, u ; u, \cdots, u)=0, \\
d_{s, s}\left(v_{1}, v_{2}, u, \cdots, u ; u, \cdots, u\right)=0
\end{gathered}
$$

from (6.4) which is satisfied by $f_{s, s}$ and by $h_{s, s}$. Hence we have obtained the following theorem.

THEOREM 6.2. An immersion $f: S^{m} \rightarrow S^{n-1}$ of order $s i s$ isometric and minimal if and only if the tensor $d_{s, s}$ defined by (6.5) satisfies (6.7) for arbitrary vectors $v_{1}, v_{2}$ of $R^{m+1}$.

As $u$ in (6.7) is the position vector of a point $p$ of $R^{m+1}$ which ranges over $S^{m},(6.7)$ is equivalent to

$$
d_{s, s}\left(v_{1}, v_{2}, w, \cdots, w ; w, \cdots, w\right)=0
$$

where $v_{1}, v_{2}, w$ are arbitrary vectors of $R^{m+1}$. We denote by $D_{s, s}$ the linear space of tensors $\hat{d}_{s, s} \in B_{s, s}$ satisfying

$$
\hat{d}_{s, s}\left(v_{1}, v_{2}, w, \cdots, w ; w, \cdots, w\right)=0
$$

for arbitrary vectors of $R^{m+1} . \quad D_{s, s}$ is nothing but the linear space $W_{2}$ of do Carmo and Wallach [1].

Remark. When $s=l, B_{s, s}$ corresponds to $S H^{l, l}$ of [1], page 61 , but we consider only real valued spherical harmonics. That $D_{s, s}$ corresponds to $W_{2}$ follows directly from Theorem 1.5 of [1] and Theorem 6.2 of the present paper. It is not immediate perhaps to prove this from the definition of $W_{2}([1]$, page 50$)$ and the definition of $D_{s, s}$. that

Since $\hat{d}_{s, s}$ is an element of $B_{s, s}$, there exists a symmetric matrix $\left[d_{B A}\right]$ such

$$
\hat{d}_{s, s}=d_{B A} H^{B} \otimes H^{A} .
$$

In order to get the compact convex body $L$ in $W_{2}$ (see [1]) we take arbitrarily an element $\hat{d}_{s, s}$ of $D_{s, s}$ and search for the range of $t$ such that there exists an isometric minimal immersion $f$ satisfying

Let us put

$$
f_{s, s}=h_{s, s}+t \hat{d}_{s, s} .
$$

$$
f_{s, s}=f_{B A} H^{B} \otimes H^{A}, \quad f_{B A}=\delta_{B A}+t d_{B A} .
$$

If $|t|$ is sufficiently small, $\left[f_{B A}\right]$ is positive and the isometric minimal immersion $f$ is full.

As $\hat{d}_{s, s}$ satisfies (6.6), we get, from (6.9), $d_{B A} h^{B} h^{A}=0$. Hence, for non-trivial $\hat{d}_{s . s}$, the largest eigenvalue $\lambda_{2}$ of $\left[d_{B A}\right]$ is positive and the least eigenvalue $\lambda_{1}$ of 
$\left[d_{B A}\right]$ is negative. Thus $\left[f_{B A}\right]$ is positive if and only if $t$ satisfies

$$
-1 / \lambda_{2}<t<-1 / \lambda_{1}
$$

and in this case the immersion $f$ is full. If $\lambda_{2} t=-1$ or $\lambda_{1} t=-1$, the immersion exists but non-full. If $\lambda_{2} t<-1$ or $\lambda_{1} t<-1$, then there exists no immersion $f$ with $h_{s, s}+t \hat{d}_{s, s}$ as the associated tensor.

Thus we have proved the following theorem.

THEOREM 6.3. Let $f$ be an isometric minimal immersion of $S^{m}$ into $S^{n-1}$. Then the associated tensor $f_{s, s}$ is expressed by $f_{s, s}=h_{s, s}+t \hat{d}_{s, s}$ where $h_{s, s}$ is the associated tensor of the standard minimal immersions and $\hat{d}_{s, s}$ is an element of $D_{s, s .} \quad t$ belongs to the interval $I\left(\hat{d}_{s, s}\right)$ defined by $\lambda_{2} t \geqq-1, \lambda_{1} t \geqq-1$ where $\lambda_{2}$ and $\lambda_{1}$ are the largest and the least eigenvalues respectively of the symmetric matrix $\left[d_{B A}\right]$ defined by $\hat{d}_{s, s}=d_{B A} H^{B} \otimes H^{A}$ and $\lambda_{1}<0<\lambda_{2}$. If $t$ is an interior point of the interval, $f$ is a full immersion. If $t$ is a boundary point of the interval, $f$ is a non-full immersion. Conversely, for any element $\hat{d}_{s, s}$ of $D_{s, s}$ and a number $t$ belonging to the interval $I\left(\hat{d}_{s, s}\right)$ stated above, there exists an equivalence class of isometric minimal immersions $f: S^{m} \rightarrow S^{n-1}$ whose associated tensor of degree $2 \mathrm{~s}$ is $h_{s, s}+t \hat{d}_{s, s}$.

\section{Isometric minimal immersions and the Gauss map.}

An isometric minimal immersion $f: S^{m} \rightarrow S^{n-1}$ of order 2 and $n=m(m+3) / 2$ gives a Veronese manifold. Properties of the Gauss map of a Veronese manifold considered as a submanifold of the Euclidean $n$-space were studied in [6]. Here we study more general cases.

$\mathbf{1}^{\circ}$. We consider isometric minimal immersions $f: S^{m} \rightarrow S^{n-1}$, an isometric embedding $i: S^{n-1} \rightarrow R^{n}$ whose image $i\left(S^{n-1}\right)$ is a hypersphere of radius $r$, and the Gauss map $\Gamma: i \circ f\left(S^{m}\right) \rightarrow G(m, n-m)$. We assume that the Grassmann manifold $G(m, n-m)$ is endowed with the standard Riemannian metric $\tilde{G}$ and the image $\Gamma\left(i \circ f\left(S^{m}\right)\right)$ is endowed with the Riemannian metric $G$ induced from $\tilde{G}$. Then we can prove the following theorem.

THEOREM 7.1. The Gauss map $\Gamma$ is homothetic, namely, the rmage $\Gamma\left(\imath \circ f\left(S^{m}\right)\right)$ is homothetic to the standard sphere $\left(S^{m}, g\right)$.

Let us choose a suitable covering $\left\{U_{\lambda}, \lambda \in \Lambda\right\}$ of $S^{m}$ by coordinate neighborhoods, namely, a covering such that, for each coordinate neighborhood $U_{\lambda}$, the image $\Gamma\left(i \circ f\left(U_{\lambda}\right)\right)$ is a coordinate neighborhood in $\Gamma\left(i \circ f\left(S^{m}\right)\right)$. Then the local coordinates $x^{1}, \cdots, x^{m}$ valid in $U_{\lambda}$ are still valid in $\Gamma\left(2 \circ f\left(U_{\lambda}\right)\right)$ and the local components $G_{\mu \lambda}$ of the Riemannian metric $G$ are given by

$$
G_{\mu \lambda}=\sum_{A} H_{\mu \rho}{ }^{A} H_{\lambda \sigma}{ }^{A} g^{\rho \sigma}
$$


where $H_{\mu \lambda}{ }^{A}$ are the local components of the second fundamental form of $i \circ f\left(S^{m}\right)$.

If $C$ is an element of $D_{s, s}$, then we have

$$
\begin{aligned}
& C(a, b, v, \cdots, v ; v, \cdots, v)=0, \\
& C(a, v, \cdots, v ; b, v, \cdots, v)=0
\end{aligned}
$$

for any vectors $a, b, v$ of $R^{m+1}$. (7.1) comes from (6.8) while (7.2) comes from (3.23) just as (6.8) came from (6.4) which is equivalent to (3.22). If $v$ is replaced by $v+c$ where $c$ is also an arbitrary vector in (7.1), we immediately get

$$
(s-2) C(a, b, c, v, \cdots, v ; v, \cdots, v)+s C(a, b, v, \cdots, v ; c, v, \cdots, v)=0
$$

which implies that $C(a, b, v, \cdots, v ; c, v, \cdots, v)$ is symmetric in $a, b, c$. On the other hand, we get, from (7.2),

$$
C(a, c, v, \cdots, v ; b, v, \cdots, v)+C(a, v, \cdots, v ; b, c, v, \cdots, v)=0 .
$$

Thus we have

$$
\begin{aligned}
& C(a, b, c, v, \cdots, v ; v, \cdots, v)=0, \\
& C(a, b, v, \cdots, v ; c, v, \cdots, v)=0 .
\end{aligned}
$$

Furthermore we get, from (7.3) and (7.4),

$$
\begin{aligned}
& (s-3) C(a, b, c, d, v, \cdots, v ; v, \cdots, v) \\
& \quad+s C(a, b, c, v, \cdots, v ; d, v, \cdots, v)=0, \\
& (s-2) C(a, b, d, v, \cdots, v ; c, v, \cdots, v) \\
& \quad+(s-1) C(a, b, v, \cdots, v ; c, d, v, \cdots, v)=0
\end{aligned}
$$

which are replaced by simpler formulas if $s=2$ or 3 . Thus we get

$$
\begin{array}{ll}
\sum_{i} C\left(e_{\imath}, a, v, \cdots, v ; e_{\imath}, b, v, \cdots, v\right)=0 & (s \geqq 2), \\
\sum_{i} C\left(e_{\imath}, a, b, v, \cdots, v ; e_{\imath}, v, \cdots, v\right)=0 & (s \geqq 3) .
\end{array}
$$

As we have (3.7) for the second fundamental form, we get, after some calculation using (2.5), (3.17),

$$
\begin{aligned}
G_{\mu \lambda}= & \left(s^{2} r^{2}+2 s(s-1) /(s+m-1)\right) g_{\mu \lambda} \\
& +s^{2}(s-1)^{2} \chi_{2,2}\left(\nabla_{\mu} u, \nabla_{\rho} u ; \nabla_{\lambda} u, \nabla_{\sigma} u\right) g^{\rho \sigma} .
\end{aligned}
$$

On the other hand, we have, in view of (3.2) and (3.18),

$$
\begin{aligned}
\chi_{2,2}\left(\nabla_{\mu} u\right. & \left., \nabla_{\rho} u ; \nabla_{\lambda} u, \nabla_{\sigma} u\right) g^{\rho \sigma} \\
= & \Sigma_{\imath} f_{s, s}\left(\nabla_{\mu} u, e_{\imath} ; \nabla_{\lambda} u, e_{\imath}\right)-s^{-2} g_{\mu \lambda},
\end{aligned}
$$


and, substituting $f_{s, s}=h_{s, s}+C$ into the right-hand-side, we get

$$
\begin{aligned}
& \chi_{2,2}\left(\nabla_{\mu} u, \nabla_{\rho} u ; \nabla_{\lambda} u, \nabla_{\sigma} u\right) g^{\rho \sigma} \\
& \quad=\sum_{i} h_{s, s}\left(\nabla_{\mu} u, e_{\imath}, u, \cdots, u ; \nabla_{\lambda} u, e_{\imath}, u, \cdots, u\right)-s^{-2} g_{\mu \lambda}
\end{aligned}
$$

by virtue of (7.7). This implies that the difference between a general isometric minimal immersion and a standard minimal immersion does not appear in the metric $G$ and we get the following lemma.

LEMMA 7.2. Let $h$ and $f$ be respectively a standard mmmal immersion and a general isometric minimal immersion, both of $S^{m}$ into $S^{n-1}$ and of the same order. Then the Gauss image of $\imath \circ f\left(S^{m}\right)$ is isometric with the Gauss image of $i \circ h\left(S^{m}\right)$.

Now we calculate

$$
h_{s, s}\left(\nabla_{\mu} u, \nabla_{\rho} u, u, \cdots, u ; \nabla_{i} u, \nabla_{\sigma} u, u, \cdots, u\right) g^{\rho \sigma} .
$$

Since $U$ is given by (4.3) and $u$ satisfies $\left\langle u, \nabla_{\lambda} u\right\rangle=0$ and $\left\langle\nabla_{\mu} u, \nabla_{i} u\right\rangle=g_{\mu \lambda}$, we immediately get

$$
U\left(\nabla_{\mu} u, \nabla_{\rho} u, u, \cdots, u ; \nabla_{\lambda} u, \nabla_{\sigma} u, u, \cdots, u\right) g^{\rho \sigma}=k_{s, m} g_{\mu \lambda}
$$

where $k_{s, n}$ is a constant depending on $s$ and $m$. As $h_{s, s}=c^{\prime} U$, this proves that the Gauss image of $i \circ h\left(S^{m}\right)$ is homothetic to the standard sphere $\left(S^{m}, g\right)$. As we have Lemma 7.2, we have proved Theorem 7.1.

$2^{\circ}$. Let $(a, b)$ be an open interval of $R$ and $f(t): S^{m} \rightarrow S^{n-1}$ be an isometric minimal immersion depending differentiably on $t$ in $(a, b)$. Then we can prove the following theorem.

THEOREM 7.3. There exist no $t$ dependent isometric minimal immersions such that the Gauss image of $i \circ f(t)\left(S^{m}\right)$ is fixed pointwise.

Deformations of an immersed submanifold of a Euclidean space such that the Gauss image is fixed pointwise are studied for example in [5]. If the Gauss image of $\imath \circ f(t)\left(S^{m}\right)$ is fixed pointwise, there exists a $(1,1)$-tensor field $a$ on $S^{m}$ whose local components $a_{\mu}^{\lambda}$ satisfy

$$
\partial B_{\lambda}^{A} / \partial t=a_{\lambda}{ }^{\sigma} B_{\sigma}^{A} .
$$

As $f(t)$ is an isometric immersion, we get, from (2.6) and $\partial g_{\mu \lambda} / \partial t=0$,

$$
a_{\mu \lambda}+a_{\lambda \mu}=0
$$

where $a_{\mu \lambda}=a_{\mu}{ }^{\sigma} g_{\sigma \lambda}$.

Now we can consider that the parameter $t$ is involved only in the tensors $F^{A}$ associated with the immersion $f(t)$, and we get, from (3.1) and (7.11), 


$$
\left(d F_{\imath_{1} \cdots \imath_{s}}{ }^{A} / d t\right) \nabla_{\lambda} u^{\imath_{1}} u^{\imath_{2}} \cdots u^{\imath_{s}}=a_{\lambda}{ }^{\sigma} F_{\imath_{1} \cdots \imath_{s}}{ }^{A} \nabla_{\sigma} u^{\imath_{1}} u^{\imath_{2}} \cdots u^{\imath_{s}} .
$$

Then applying the operator $\nabla_{\mu}$ we get

$$
\begin{aligned}
& -g_{\mu \lambda}\left(d F_{\imath_{1} \cdots u_{s}}{ }^{A} / d t\right) u^{\imath_{1}} \cdots u^{\imath_{s}} \\
& +(s-1)\left(d F_{\imath_{1} \cdots \imath_{s}}{ }^{A} / d t\right) \nabla_{\mu} u^{\imath_{1}} \nabla_{\lambda} u^{\imath_{2}} u^{\imath_{3}} \cdots u^{\imath_{s}} \\
& =F_{\imath_{1} \cdots \imath_{s}}{ }^{A}\left(\nabla_{\mu} a_{\lambda}{ }^{\sigma} \nabla_{\sigma} u^{\imath_{1}} u^{\imath_{2}} \cdots u^{\imath_{s}}-a_{\lambda \mu} u^{\imath_{1}} \cdots u^{\imath_{s}}\right.
\end{aligned}
$$

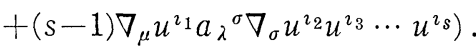

As we have $\nabla_{\mu} a_{\lambda}{ }^{\kappa}=\nabla_{\lambda} a_{\mu}{ }^{\kappa}$ by virtue of (7.11), we get

$$
\begin{aligned}
& \left(a_{1 \ell \lambda}-a_{\lambda \mu}\right) F_{\imath_{1} \cdots \imath_{s}}{ }^{A} u^{\imath_{1}} \cdots u^{\imath_{s}}
\end{aligned}
$$

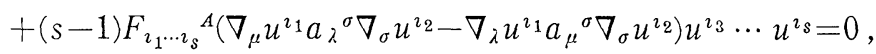

hence

$$
\begin{aligned}
& \left(a_{\mu \lambda}-a_{\lambda \mu}\right) f_{s, s}(u, \cdots, u ; u, \cdots, u) \\
& \quad+(s-1)\left(a_{\lambda}{ }^{\sigma} \chi_{2}\left(\nabla_{\mu} u, \nabla_{\sigma} u\right)-a_{\mu}{ }^{\sigma} \chi_{2}\left(\nabla_{\lambda} u, \nabla_{\sigma} u\right)\right)=0 .
\end{aligned}
$$

Substituting $f_{s . s}(u, \cdots, u ; u, \cdots, u)=r^{2}=m /(s(s+m-1))$ and (3.17) into this equation, we get

$$
s^{-1}\left(a_{\mu \lambda}-a_{\lambda \mu}\right)=0 \text {. }
$$

Thus we get $a_{\mu \lambda}=0$, hence

$$
\left(d F_{\imath_{1} \cdots \imath_{s}}{ }^{A} / d t\right) \nabla_{\lambda} u^{\imath_{1}} \mathcal{u}^{\imath_{2}} \cdots u^{\imath_{s}}=0
$$

from (7.13). Consequently the $n$ functions

$$
\left(d F_{\imath_{1} \cdots \imath_{s}}{ }^{A} / d t\right) u^{\imath_{1}} \cdots u^{\imath_{s}}=\phi^{A}(t)
$$

can depend only on $t$. On the other hand, as we have

$$
\sum_{\imath} d F_{i i i_{3} \cdots \tau_{s}}{ }^{A} / d t=0 \text {, }
$$

$\phi^{A}(t)$ are eigenfunctions of order $s$, hence $\phi^{A}(t)=0$. Thus we have proved the theorem.

$3^{\circ}$. The present author studied Gauss-critical immersions and Gauss-critical submanifolds in [4]. If $\imath$ is an immersion of a compact orientable $C^{\infty}$ manifold $M$ of dimension $m$ into the Euclidean $n$-space $R^{n}$ and the Gauss map $\Gamma: i M \rightarrow$ $G(m, n-m)$ is regular, then the submanifold $\imath M$ of $R^{n}$ and the submanifold $\Gamma(i M)$ of the Grassmann manifold $G(m, n-m)$ can be considered as Riemannian manifolds $\left(i M, g_{\imath}\right)$ and $\left(\Gamma(i M), G_{\imath}\right)$ respectively where $g_{\imath}$ and $G_{i}$ are Riemannian metrics induced from the standard metrics of $R^{n}$ and of $G(m, n-m)$ respectively. Let us assume that $\left\{U_{\lambda}, \lambda \in \Lambda\right\}$ is a suitable covering of $M$ by coordinate neighborhoods such that $\left\{\Gamma\left(i U_{\lambda}\right), \lambda \in \Lambda\right\}$ is a covering of the image manifold $\Gamma(i M)$ by coordinate neighborhoods so that we can use $x^{1}, \cdots, x^{m}$ as local coordinates 
of $M$ and of $\Gamma(i M)$ simultaneously. Using such local coordinates, we see that the local components of $g_{\imath}$ and of $G_{\imath}$ are written $g_{\mu \lambda}$ and $G_{\mu \lambda}$ respectively. Then let us define $\mathrm{Vol}^{*}\left(\Gamma(i M), G_{\imath}\right)$ by

$$
\mathrm{Vol}^{*}\left(\Gamma(i M), G_{\imath}\right)=\int_{M}\left(\operatorname{det}\left[G_{\mu \lambda}\right] / \operatorname{det}\left[g_{\mu \lambda}\right]\right)^{1 / 2} \mu\left(g_{\imath}\right)
$$

where $\mu\left(g_{\imath}\right)$ is the volume form of $\left(i M, g_{\imath}\right)$. A Gauss-critical immersion $\imath_{c}$ is defined as an immersion which is a critical point in $\mathcal{I}_{M}$ of the integral $\operatorname{Vol}^{*}\left(\Gamma(i M), G_{\imath}\right)$ where $\mathcal{G}_{M}$ is the space of immersions $\imath$ of $M$ such that $\Gamma(i M)$ is regular. The resulting submanifold $i_{c} M$ is called a Gauss-critical submanifold. Theorem 4.4 of [4] states the following result.

If $\imath: M \rightarrow R^{n}$ is an immersion such that $\left(\imath M, g_{\imath}\right)$ is an Einstein manifold, $\Gamma: \imath M \rightarrow G(m, n-m)$ is a homothetic mapping and moreover the components of the mean curvature vector of $\imath M$ are eigenfunctions of the Laplacian on $\left(\imath M, g_{\imath}\right)$ belonging to an eigenvalue, then $\imath$ is a Gauss-critical immersion.

In our case, $\left(\imath M, g_{\imath}\right)$ is $i \circ f\left(S^{m}\right)$ endowed with the Riemannian metric isometric to the standard sphere $\left(S^{m}, g\right)$, hence an Einstein manifold, the Gauss map is homothetic and

$$
\frac{1}{m} g^{\mu \lambda} H_{\mu \lambda}{ }^{A}=-\frac{s(s+m-1)}{m} f^{A} .
$$

Hence $i \circ f\left(S^{m}\right)$ is a Gauss-critical submanifold.

From (7.14) we immediately find that the mean curvature vector of $\imath \circ f\left(S^{m}\right)$ is parallel in the normal bundle.

Thus we have proved the following theorem (see also [8], [6]).

THEOREM 7.4. Let $f: S^{m} \rightarrow S^{n-1}$ be an isometric minimal immersion and $i: S^{n-1} \rightarrow R^{n}$ be an isometric embedding so that the image is a hypersphere of radius $r$. Then $i \circ f\left(S^{m}\right)$ is a Gauss-critical submanıfold and its Gauss map is harmonic.

\section{REFERENCES}

[1] M. P. Do CARMo And N.R. Wallach, Minimal immersions of spheres into spheres, Ann. of Math., (2) 93 (1971), 43-62.

[2] T. Itoh AND K. Ogiue, Isotropic immersions and Veronese manifolds, Trans. Amer. Math. Soc., 209 (1975), 109-117.

[3] K. Mashimo, Minimal immersions of 3-dimensıonal sphere into spheres, Osaka Math. J. (to appear).

[4] Y. Mutō, The Gauss map of a submanifold in a Euclidean space, J. Math. Soc. Japan, 30 (1978), 85-100.

[5] Y. Mutō, Deformability of a submanifold in a Euclidean space whose image by the Gauss map is fixed, Proc. Amer. Math. Soc., 76 (1979), 140-144.

[6] Y. Mutō, Submanifolds of a Euclidean space with homothetic Gauss map, J. Math. Soc. Japan, 32 (1980), 531-555. 
[7] H. Nakagawa and T. ITOH, On isotropic Immersions of space forms into a space form, Minimal submanifolds and geodesics, Kargai Publications, Tokyo, 1978, 255-271.

[8] E.A. Ruh AND J. Vilms, The tension field of the Gauss map, Trans. Amer. Math. Soc., 149 (1970), 569-573.

[9] T. Takahashi, Minimal immersions of Riemannian manifolds, J. Math. Soc. Japan, 18 (1966), 380-385.

[10] K. TsukadA, Isotropic minimal immersions of spheres into spheres, J. Math. Soc. Japan, 35: (1983), 355-379.

2262-150, ТомIOKA-CHO

KanazaWA-KU, YoKoHama

236, JAPAN 